\title{
The Application of Topological Structure of Interpersonal Relationships to Optimize Customer Segmentation
}

\author{
Haotian Liu \\ Rutgers University, Rutgers Business School, Newark, United State, 07103 \\ ${ }^{*}$ Corresponding author. Email: janeliu0528@gmail.com
}

\begin{abstract}
Customer segmentation is based on a large amount of customer data, but for many new users, their customer data is zero. The similarity of the characteristics of the interpersonal relationship group can make up the shortcomings of the existing customer segmentation method, so this topic is studied. This article improves the accuracy and coverage of customer segmentation in the current main customer segmentation methods. Through the combination with topological structure, we divided customer groups by using interpersonal relationship. The method not only provided a more accurate customer segmentation method, but also established a database for potential customers to ensure that potential customers receive the best service from the first time of shopping. What's more, the combination of existing customer segmentation method and topology structure provides a more comprehensive and accurate information foundation for enterprises to study customer data. For the unoptimized vulnerability in this method, that is, how to determine the percentage of selection of primary user, further research will be carried out in the future. This improvement has vital significance for companies to expand their customer base and increase sales and profit.
\end{abstract}

Keywords: Topology, customer segmentation, interpersonal relationship, potential customer, business analytics

\section{INTRODUCTION}

At the beginning of the formation of the commodity market, commodities were only distinguished by simple categories, such as milk, furniture, cups, and pots. But with the continuous development of the commodity economy, some consumers gradually have higher requirements for the quality of life. At the same time, combined with the in-depth research in other fields, people are gradually refined commodity categories according to factors such as raw materials and processes, such as, skimmed milk, high-calcium milk, vitamin D Milk. Consumers often can't make the best choice when they are shopping, because they know too little knowledge about that kind of product. Therefore, the concept of "market segmentation" is proposed, and the main purpose of segmentation is the concentration of marketing energy and force on the subdivision to gain a competitive advantage within the segment. [1]

The current common customer segmentation method is to use cluster analysis to get the characteristics of similar consumers, but this analysis mode is mostly applicable to existing customers. When a potential customer turns into a new customer, there is no consumption record data, or there is too little data, which makes the company unable to make a suitable recommendation for the first-time consumer. This kind of information gap will cause consumers and companies to be unable to meet their respective needs. In order to achieve a win-win situation, it is important to study how the topological structure of consumer relationships can be used to make up for the missing information and improve the current customer segmentation method.

This article will start with the cluster analysis method commonly used in the current customer segmentation method and analyze its advantages and disadvantages. Then introduce how to define the topological structure network of consumer interpersonal relationship, and how to apply it in the current analysis process. Finally, this article will present DIDI as an example to introduce how to apply it in enterprises and how to improve it. 


\section{DEFINITION, PROCESS AND APPLICATION}

\subsection{Method of Customer Segmentation}

Segmentation was introduced by Wendell R. Smith in 1956. In his opinion, segmentation is based upon developments on the demand side of the market and represents a rational and more precise adjustment of product and marketing effort to consumer or user requirements. [2] Meeting demand means improving the quality of products and services, and customer segmentation means providing different services to customers with different needs. Therefore, how to make more reasonable customer segmentation has become a constant subject of research. The current common segmentary methods mainly include single variable method, principal factor method, multi-variable segmentary method, series factor segmentary method and so on. [3] The single-variable method has a relatively simple segmentary method, and each layer has a large number of customers, and this method is an early customer segmentary method. With the continuous development of computer technology, companies use computer build models to analyze consumer data in multiple dimensions, which is the multi-variable segmentary method. The multi-variable segmentary method is based on a large amount of consumer information data, and extracts certain types of variables which are applicable to the corresponding product field to further segment customers. Take the clothing industry as an example. The main variables for customer segmentation in the clothing industry are usually gender, age, and income. Using these three types of factors to segment consumers in the clothing industry, the company can divide customers into 18 groups. (Figure 1) Compared to dividing into two or three customer groups based on a single variable such as gender or age, the multi-dimensional analysis method provides more accurate customer segmentation, and then further improves the accuracy of marketing plans. In addition to the above approaches for customer segmentation based on characteristics that are already known, machine learning provides technical support for optimizing segmentation basing on mathematical methods to analyze customer characteristics. Cluster analysis is widely used. Through mathematical calculation methods, the data is optimized segmented, and then the consumer characteristics are concluded by analyzing the consumption data of each customer group. Finally, the characteristics of each customer group are defined. (Figure 2)

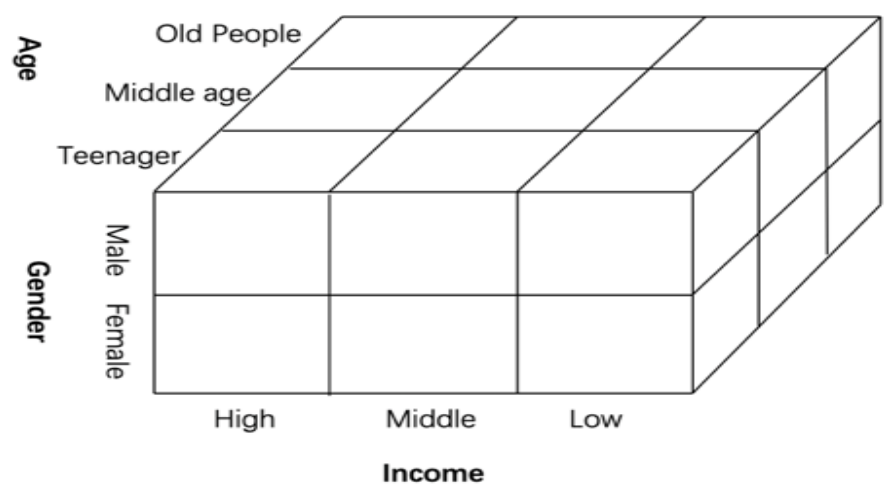

Figure 1. Multi-variable segmentation for clothing industry

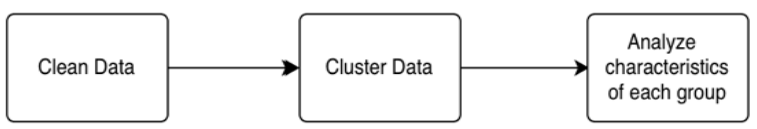

Figure 2. Process of cluster analysis

Customer segmentation can objectively reflect the common characteristics of the customer group and comprehensively show the understanding of the various characteristics of customers, which is conducive to marketers to understand customer value in a more in-depth and detailed manner, and facilitate the dynamic tracking and maintenance of customers. [4] Through multi-dimensional analysis of user characteristics, companies can more accurately conclude consumers' behavior habits and consumption preferences. This is very important to the improvement of the service quality of the company. Although customer segmentation has been greatly improved on the basis of the original segmentary method, and the method becomes more accurate, these methods still have some defects. 
Although the existing consumer segmentation methods can analyze consumer behaviors to the maximum extent and divide them accurately into groups based on different characteristics. The premise for accurate consumer analysis is that the analyzed consumer must have data of previous consumer behaviors and certain amount of consumption data. For potential consumers, they have never had consumption behavior, so there is no consumption data. If such consumers are unwilling to provide personal information and do not have consumption data, and then the company doesn't have any basic data of such users. Finally, these customers may have a greater chance to give up continuing to consume on this company or platform. Increasing the probability of successfully converting potential customers with "zero consumption data" into new customers will become an urgent problem for enterprises. An effective solution to this problem will maximize the scale of users and increase the profits of enterprises or platforms.

\subsection{Method of Topology}

The topological structure is mostly used in the construction of computer networks. According to its

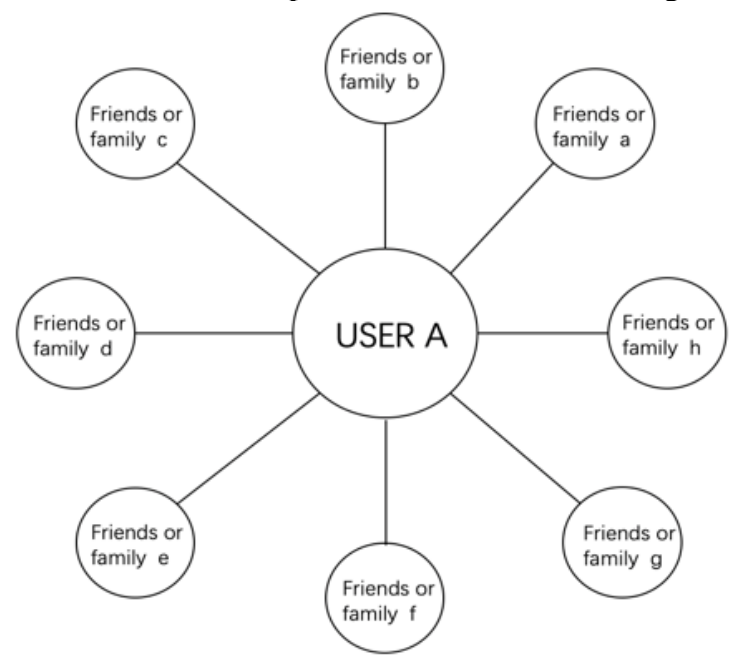

connection and node connection mode, it can be divided into the following types: (1) bus type, (2) ring, (3) star, (4) Net type. [5] The star topology has the advantages of simple structure and easy management, and it is similar to the interpersonal relationship diagram. Interpersonal relationship refers to the psychological relationship and psychological distance that people form in the process of interpersonal communication. Harmony or disharmony, mutual attraction or mutual repulsion between the two parties in personalities, attitudes, emotions, etc. will inevitably lead to intimacy or alienation in their interpersonal relationship. [6] Therefore, using the topological structure diagram of interpersonal relationships can analyze the similar characteristics of the groups in this relationship network and be the supplement of incomplete personal characteristics of consumers in that network. The information obtained by categorizing and summarizing the characteristics in the interpersonal structure network can provide some effective characteristics of the new user who is on the basis of "zero data".

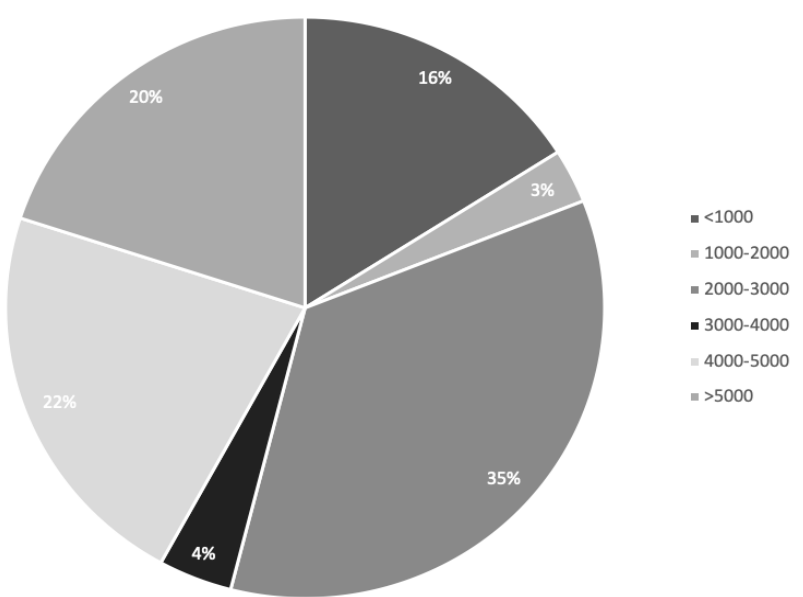

Figure 3. Topological structure of interpersonal relationships Figure 4. Percentage of customers with monthly income

In this part, the customer segmentation process combined with the topology of the interpersonal relationship will be presented. First, collect user address book data and interaction data. Through the random selection system, a certain percentage of the existing customers are taken as the main users of the topology center, and the interpersonal relationship topological diagrams are constructed for these customers. Taking the main user $\mathrm{A}$ as an example. Friends or family member a, b, c, d, e, f... are all nodes in the topological structure of their interpersonal relationships. (Figure 3) Organize and analyze the personnel information in this topology. For the first step, sort out the people who exist in the address book of user A but do not exist in this enterprise user group, and store such people in the "potential user" database. Secondly, the mode and average statistics are performed on the information of other people in this topology, and then obtain the common characteristics of such users. The obtained characteristic information will be filled into the information column of the potential user accordingly, so that when the potential customer performs his or her first shopping behavior, the database of the company can provide targeted services based on the known information. In addition, companies can also use this topology to perform the initial segmentation of users on the basis of more complete and accurate information and data. The accuracy of using the mode or average of the overall data to fill in missing values is much lower than the accuracy of supplementary data obtained by using 
interpersonal groups. At the same time, it can also be found which company's customer groups are missing based on statistical customer database data. For example, in the customer database, the percentage of customers with monthly income above 5000 dollars accounts for $20 \%$, the percentage of customers earning 2000 to 3000 dollars accounts for $35 \%$, but the percentage of people earning 1,000 to 2,000 accounts only $3 \%$, and the percentage of people earning 3000 to 4000 accounts only $4 \%$. Then companies need to investigate these groups of customers, and find out the reasons for the low percentage and rise appropriate solutions. (Figure 4)

The combination of interpersonal relationship topology and cluster analysis method can achieve full coverage of potential customers and existing customers. This method not only can provide customized services for customers on a larger scale, but also improve the effectiveness of customer segmentary groups. First of all, this method can improve the current data analysis process, increase many different means to obtain user data, and use the common characteristics of interpersonal relationships to fill the blank information of new users and provide targeted services for them. What's more, this method will greatly improve the initial experience satisfaction of new users, thereby increasing the user conversion rate, and also provide a larger data basis for the subsequent analysis process. Secondly, if the user conversion rate is improved, then this represents that the company's income will increase. With the overall cost remaining stable, if the company's total sales continue to rise, it means that the company's profits will rise. For enterprises, profit is the ultimate goal of business operations. Finally, based on the interpersonal relationship of users, group consumption patterns such as consumer coupons and family cards can be introduced accordingly to increase user stickiness and extend the life cycle of user value.

\subsection{Application}

Didi Chuxing ("DiDi") is the world's leading mobile transportation and convenience platform. The company offers a full range of app-based transportation and life services for over 550 million users across Asia, Latin America and Australia, including Taxi, Express, Premier, Luxe, Bus, Designated Driving, Enterprise Solutions, Bike Sharing, E-bike Sharing, Automobile Solutions, Food Delivery and Payment. [7] The current data analysis mode of DIDI is to collect data from services that the customers use and analyze the characteristics of customers' performances. This approach has certain drawbacks as mentioned above.

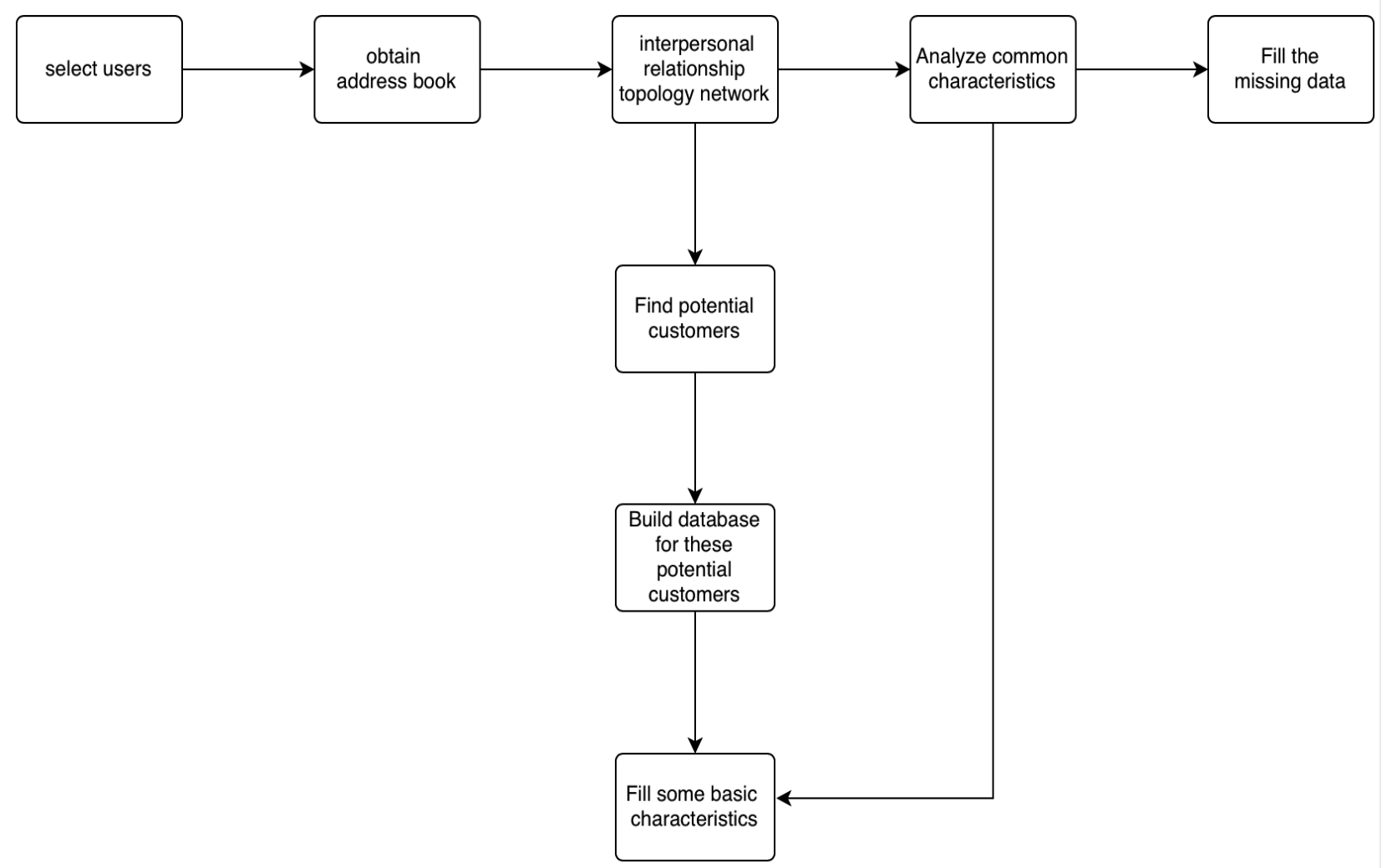

Figure 5. Process of topological analysis for DID

However, through the acquisition of the user's address book and the collection and analysis of the user's interpersonal network data, it is possible to know the group characteristics of users in the interpersonal 
network. Therefore, we will establish an address book function for DIDI. Users can also add their family and friends to the DIDI address book. Regarding the choice of the main user, which is the user in the center of interpersonal relationship topology network, we will use a random selection method to select $30 \%$ of the users as the main users. In addition, after the user uses the service of helping others to call a car, the address book will automatically add the other person to their address books. In addition, before a potential user uses the service for the first time, the company can recommend targeted services and products based on the group characteristics of the user's network. If the analysis shows that certain types of users are more frequent to help others to call a car, then group coupons can be recommended for such users, so that more users can join this customer group, and it is of vital importance of new users' expansion. (The process is shown in Figure 5)

Using the interpersonal relationship topology into the process of DIDI's analysis to obtain information can not only expand DIDI's new customer base, but also fill in missing information to the greatest extent, improve the accuracy of subsequent user behavior analysis, and do better customer segmentation. The introduction of group coupons will also increase user retention, extend the life cycle of DIDI users, and create greater user value for DIDI.

In the application of this model, further simulations and experiments are needed for the part of percentage of the main users' selection. Through continuous experimentation, it should be determined how to choose the number of selected main users is sufficient to cover all users and will not cause excessive overlap. In the current research, there is only the idea put forward and there are no corresponding experiments and modeling. This question should be solved in future researches

\section{CONCLUSION}

This article analyzes and complements customer information by using the topological structure of interpersonal relationships and the sociological characteristics of interpersonal relationship groups, and adds the completed information into the existing customer segmentation method. This method can maximize the degree of completion of customer information and data, and make business analysis results more credible. At the same time, the information obtained through the topological structure of interpersonal relationships can be applied to the customer segmentation of new users for the first time to achieve full coverage of user analysis, and provide high quality services to potential customers. However, the method of determining the number of main users still needs further research.

\section{ACKNOWLEDGMENT}

It took me a month to write this paper. Thanks to Professor Stephen Coggeshall, I got a lot of inspiration for this paper from his course. I also thank my instructor for giving me a lot of help in the whole process from selecting the topic to checking the information and revising the paper. Due to my limited level of theoretical knowledge, the opinions and application explanations may have some omissions and deficiencies. Welcome criticism and corrections from teachers and experts.

\section{REFERENCES}

[1] Jerry W. Thomas. Market Segmentation. Decision Analyst. 1.817.640.6166. 2019

[2] Wendell R. Smith.. Product Differentiation and Market Segmentation As Alternative Marketing Strategies. Journal of Marketing. Page 3-8.1956

[3] Jin Rungui. Marketing Third Edition. Higher Education Press.2010.8

[4] Qianqian Zheng. Research on Customer Segmentation Based on Data Mining. TP311.13. 2013

[5] Fei Nie. Talking about the Topological Structure of Computer Network [J]. Heilongjiang Science and Technology Information. 2010(03):66.

[6] Shi Ronghua. Social Psychology Dictionary: Sichuan People's Publishing House. 1988-11.

[7] About us. https://www.didiglobal.com 